\title{
Spinning string fluid dynamics in general relativity
}

\author{
Larry L Smalley† and Jean P Krisch $\ddagger$ \\ $\dagger$ Department of Physics, University of Alabama in Huntsville, Huntsville, AL 35899, USA \\ $\ddagger$ Department of Physics, University of Michigan, Ann Arbor, MI 48109, USA
}

Received 9 June 1997

\begin{abstract}
The general, energy-momentum tensor for a dynamical, spinning string fluid in general relativity is presented using the Ray-Hilbert variational principle. The calculations are given for both the standard and the extended thermodynamics versions in which the latter includes both the spin and string as thermodynamic variables. Both versions yield the same Fermi-Walker transport of the spin with a correction term due to the string. As an unexpected general feature, it is shown that the string and spin are dual 2-forms. Examples of solutions to the field equations are given for the extension of static black holes for non-spinning, string fluids to stationary, slowly rotating black holes for spinning string fluids. These solutions are then compared with solutions obtained from the postulated energy-momentum tensor of Letelier. The general feature of these solutions for positive density is that the spin causes the event horizon for the stationary black hole to contract whether or not the spin is considered as a thermodynamic variable.
\end{abstract}

PACS numbers: $0440 \mathrm{~N}, 0570,9880 \mathrm{H}$

\section{Introduction to spinning string fluids in general relativity}

The discussion of the cosmological implications of strings goes back nearly 20 years to the work of Zeldovich [1] and Vilenkin [2]. A string dust model was introduced by Letelier [3] from the description of the string function as a spacetime, surface-forming bi-vector

$$
\Sigma^{\mu \nu}=\epsilon^{A B} \frac{\partial x^{\mu}}{\partial \lambda^{A}} \frac{\partial x^{v}}{\partial \lambda^{B}}
$$

where

$$
\epsilon^{A B}=\left\{\begin{array}{lll}
1 & \text { if } \quad A=0, & B=1 \\
-1 & \text { if } \quad A=1, & B=0 \\
0 & \text { if } \quad A=B . &
\end{array}\right.
$$

In a previous work on the dynamics of a string fluid [4], it was noted that the form of equation (1) follows closely the form of the spin bi-vector developed by Halbwachs [5], and therefore the description of a string fluid energy density will use the parallel description of the velocity matrix used in discussions of fluid dynamics in continuum mechanics (see, for example, the discussions of fluids with spin and twist in metric affine geometry for a more complete discussion [6]). The basic discussion of string fluid dynamics plus applications to static black hole models and cosmological solutions in general relativity (GR) are described by [7] (referred to as paper I). In this work, a much more extensive discussion extends the concept of the fluid dynamics of strings to the case of spinning string fluids. This means that 
in a fluid context, a Ray-Hilbert variational principle [8] can be developed for a spinning string fluid by introducing a complete set of tetrads for the fluid

$$
\Sigma^{i j}=\rho \lambda(x)\left(a^{4 i} a^{3 j}-a^{4 j} a^{3 i}\right) \doteq \rho \sigma^{i j}
$$

along with the usual spin density

$$
S^{i j}=\rho k(x)\left(a^{1 i} a^{2 j}-a^{1 j} a^{2 i}\right) \doteq \rho s^{i j}
$$

where $\rho$ is the string density, $a^{\mu i}$ are the tetrads, where the Latin indices, $i=0,1,2,3$, are the holonomic coordinates, the Greek indices, $\mu=1,2,3,4$ are the non-holonomic coordinates, and where $k(x)$ and $\lambda(x)$ are the spin and string (module) functions, respectively (considered as a parametric function), which will not be varied directly. The holonomic metric has signature, $g_{i j}=(-1,1,1,1)$, and the non-holonomic metric, $\eta_{\mu \nu}=(1,1,1,-1)$. We also identify the tetrad $a^{4 i} \equiv u^{i}$. Using equations (3) and (4), the equivalent 'angular' velocity matrix takes the form

$$
\omega^{i j}=\dot{a}^{\alpha i} a_{\alpha}{ }^{j}
$$

so that the string energy density is given by

$$
T_{S T}=\frac{1}{2} \Sigma^{i j} \omega_{i j}=\rho \lambda a^{4}{ }_{i} \dot{a}^{3 i}
$$

in comparison with the spin energy density

$$
T_{S}=\frac{1}{2} S^{i j} \omega_{i j}=\rho \lambda a^{1}{ }_{i} \dot{a}^{2 i} .
$$

Some additional justification for this string formulation is given by Nieto [9]. It is interesting to note the similarity of the spin/string energy density in the fluid framework to that which occurs in general spin/twist formulations [6].

In a previous work [4], the generality of the spacetime manifold was not restricted to the Riemannian geometry of GR. However, this work will restrict itself to the Riemannian manifold since the examples discussed in the penultimate section are compared to the behaviour of strings in general relativity (GR).

In the next section, the treatments of spinning string fluids using both the improved and unimproved thermodynamic versions are described. Examples of spinning string solutions are given in section 3 , and the conclusions and discussions are given in the final section.

\section{Spinning string fluid Lagrangian}

The Ray-Hilbert Lagrangian for a spinning string fluid in GR spacetime becomes

$$
\mathcal{L}_{\mathcal{G}}=\mathcal{L}_{\mathcal{M}}+\mathcal{L}_{\mathcal{G}}
$$

where $\mathcal{L}_{\mathcal{M}}$ is the matter Lagrangian

$$
\begin{gathered}
\mathcal{L}_{\mathcal{M}}=e\left\{-\rho[1+\epsilon(\rho, s)]+\lambda_{\mu \nu}\left(a^{\mu i} a^{\nu j} g_{i j}-\eta^{\mu \nu}\right)+\lambda_{2} \nabla_{i}\left(\rho u^{i}\right)\right. \\
\left.+\lambda_{3} u^{i} X_{, i}+\lambda_{4} u^{i} s_{, i}-k \rho a^{1 i} \dot{a}_{i}^{2}-\rho \lambda a^{4}{ }_{i} \dot{a}^{3 i}\right\}
\end{gathered}
$$

and $\mathcal{L}_{\mathcal{G}}$ is the gravitational field Lagrangian

$$
\mathcal{L}_{\mathcal{G}}=\frac{e R}{2 \kappa}
$$

where $e=\sqrt{-g}, \kappa=8 \pi G, R$ is the Riemann scalar in GR spacetime, $\lambda_{\mu \nu}$ are the Lagrange multipliers and we write $\lambda_{44}=\lambda_{1}$ in the variation. Note that all components of the tetrads occur in the Lagrangian (9), and are therefore active in the variational equations. In the first part of this section, the spin and string variables are not included in the thermodynamics 
as in the non-improved energy-momentum formulations of spinning fluids [11]. In the following abbreviated subsection, the spin and string variables are included in the extended thermodynamics as in the improved energy-momentum formulations of spinning fluids [12].

\section{Development of the energy-momentum tensor}

\subsection{Standard thermodynamics}

The standard thermodynamics for a perfect fluid for a Ray-Hilbert [8] variational principle is given by

$$
\mathrm{d} \epsilon=T \mathrm{~d} s+\frac{p}{\rho^{2}} \mathrm{~d} \rho .
$$

The variational variables are then $g_{i j}, \rho, s, X, a^{\alpha i}$ and the various Lagrange multipliers.

\subsubsection{Variational equations.}

(i) Tetrad and density variational equations. The variational equations become:

Density variation $\delta \rho$ :

$$
-\left(1+\epsilon+\frac{p}{\rho}\right)-\dot{\lambda}_{2}-\lambda u^{i} \dot{a}_{i}^{3}-k a^{i k} \dot{a}_{k}^{2}=0 .
$$

4-velocity $\delta u$ :

$$
\begin{gathered}
-\rho \nabla_{i} \lambda_{2}+\lambda_{3} X_{, i}+\lambda_{4} s_{, i}+2 \lambda_{1} u_{i}+2 \lambda_{41} a^{1}{ }_{i}+2 \lambda_{42} a^{2}{ }_{i}+2 \lambda_{43} a^{3}{ }_{i} \\
-\rho \lambda \dot{a}^{3}{ }_{i}-\rho \lambda u_{k} \nabla_{i} a^{3 k}-\rho k a^{1 k} \nabla_{i} a^{2}{ }_{k}=0 .
\end{gathered}
$$

Tetrads

$$
\begin{array}{ll}
\delta a^{1 i}: & 2 \lambda_{11} a_{i}{ }_{i}+2 \lambda_{12} a^{2}{ }_{i}+2 \lambda_{13} a^{3}{ }_{i}+2 \lambda_{41} u_{i}-\rho k \dot{a}^{2}{ }_{i}=0 \\
\delta a^{2 i}: & 2 \lambda_{22} a^{2}{ }_{i}+2 \lambda_{12} a^{1}{ }_{i}+2 \lambda_{23} a^{3}{ }_{i}+2 \lambda_{42} u_{i}+\rho u^{k} \nabla_{k}\left(k a_{i}{ }_{i}\right)=0 \\
\delta a^{3 i}: & 2 \lambda_{33} a^{3}{ }_{i}+2 \lambda_{13} a^{1}{ }_{i}+2 \lambda_{23} a^{2}{ }_{i}+2 \lambda_{43} u_{i}+\rho u^{k} \nabla_{k}\left(\lambda u_{i}\right)=0 .
\end{array}
$$

The tetrad and density variations reveal the following relevant results for the Lagrange multipliers which will be useful in the analysis of the metric field equations and the transport properties of the spinning string.

$$
\begin{aligned}
& 2 \lambda_{1}=[\rho(1+\epsilon)+p]-T_{S T} \\
& -\rho \dot{\lambda}_{2}=[\rho(1+\epsilon)+p]+T_{S T}+T_{S} \\
& \lambda_{12}=0 \\
& \dot{k}=0 \\
& 2 \lambda_{41}=\rho k \dot{u}_{k} a^{2 k} \\
& 2 \lambda_{42}=-\rho k \dot{u}_{k} a^{1 k} \\
& 2 \lambda_{43}=-\rho \dot{\lambda} \\
& \lambda_{11} a^{1 i} a^{1 j}+\lambda_{22} a^{2 i} a^{2 j}+\lambda_{33} a^{3 i} a^{3 j}+\lambda_{13} a^{(1 i} a^{3 j)}+\lambda_{23} a^{(2 i} a^{3 j)}+\lambda_{41} u^{(i} a^{1 j)}+\lambda_{42} u^{(i} a^{2 j)} \\
& \quad+\lambda_{43} u^{(i} a^{3 j)}-\frac{1}{2} \rho k \dot{a}^{(2 i} a^{1 j)}+\frac{1}{2} \rho k \dot{a}^{(1 i} a^{2 j)}+\frac{1}{2} \rho u^{k} \nabla_{k}\left[\lambda u^{(i}\right] a^{3 j)}=0 \\
& \lambda_{41} u^{(i} a^{1 j)}+\lambda_{42} u^{(i} a^{2 j)}=\frac{1}{2} u^{(i} S^{j) k} \dot{u}_{k} \\
& \lambda_{43} u^{(i} a^{3 j)}=-\frac{1}{2} \rho \dot{\lambda} u^{(i} a^{3 j)} .
\end{aligned}
$$


Adding equations (24)-(26) gives the useful result necessary to resolve the metric field equation

$$
\begin{aligned}
\lambda_{11} a^{1 i} a^{1 j}+ & \lambda_{22} a^{2 i} a^{2 j}+\lambda_{33} a^{3 i} a^{3 j}+2 \lambda_{13} a^{(1 i} a^{3 j)}+2 \lambda_{23} a^{(2 i} a^{3 j)}+2 \lambda_{41} u^{(i} a^{1 j)} \\
& +2 \lambda_{42} u^{(i} a^{2 j)}+2 \lambda_{43} u^{(i} a^{3 j)} \\
= & \frac{1}{2} \rho k \dot{a}^{(2 i} a^{1 j)}+\frac{1}{2} \rho k \dot{a}^{(1 i} a^{2 j)}-\frac{1}{2} \rho u^{k} \nabla_{k}\left[\lambda u^{(i}\right] a^{3 j)}+\frac{1}{2} u^{(i} S^{j)} \dot{u}_{k}-\frac{1}{2} \rho \dot{\lambda} u^{(i} a^{3 j)}
\end{aligned}
$$

The final set of identities in this section comes from the antisymmetric combination of the tetrad variational equations similar to equations (24) and (25),

$$
\begin{aligned}
& 2 \lambda_{41} u^{[i} a^{1 j]}+2 \lambda_{42} u^{[i} a^{2 j]}+\rho k \dot{a}^{[1 i} a^{2 j]}-\rho k \dot{a}^{[2 i} a^{1 j]}+\rho \lambda \dot{u}^{[i} a^{3 j]}=0 \\
& 2 \lambda_{41} u^{[i} a^{1 j]}+2 \lambda_{42} u^{[i} a^{2 j]}=\dot{u}_{k} u^{[i} S^{j] k}
\end{aligned}
$$

and since $\dot{k}=0$

$$
\rho \dot{s}^{i j}=2 \rho k\left[\dot{a}^{[1 i} a^{2 j]}+a^{[1 i} \dot{a}^{2 j]}\right] .
$$

In addition, we note the obvious identity

$$
\rho \lambda \dot{u}^{[i} a^{3 j]}=u_{k} \dot{u}^{[i} \Sigma^{j] k} \text {. }
$$

Finally, combining equations (28)-(31) gives

$$
\rho \dot{s}^{i j}+2 u^{[i} S^{j] k} \dot{u}_{k}+2 \dot{u}^{[i} \Sigma^{j] k} u_{k}=0
$$

which represents the generalization of Fermi-Walker transport for the spinning string.

The variations have been made assuming that the spin vector and string vector are both along the same tetrad. With this assumption, the spin module function $k(x)$ is constant along the flow lines equation (20). If a different choice is made, for example, the string vector is written with the $e^{1 i}$ tetrad, then the dot derivative of the spin module function is proportional to the $\lambda_{12}$ multiplier and is not necessarily zero. The transport equation, as written in equation (32), is the same in both cases and therefore independent of the calculation's choice of tetrads. This results also follows for the metric field equations discussed in the next subsection.

(ii) Metric field equation. The variation with respect to the metric $g_{i j}$ gives the metric field equation

$$
\begin{aligned}
-\frac{1}{2 \kappa} G^{i j}-\frac{1}{2} \rho & (1+\epsilon) g^{i j}-\frac{1}{2} \dot{\lambda}_{2} g^{i j}+\lambda_{11} a^{1 i} a^{1 j}+\lambda_{12} a^{(1 i} a^{2 j)}+\lambda_{22} a^{2 i} a^{2 j} \\
& +\lambda_{33} a^{3 i} a^{3 j}+2 \lambda_{13} a^{(1 i} a^{3 j)}+2 \lambda_{23} a^{(2 i} a^{3 j)}+2 \lambda_{41} u^{(i} a^{1 j)}+2 \lambda_{42} u^{(i} a^{2 j)} \\
& +2 \lambda_{43} u^{(i} a^{3 j)}+\lambda_{1} u^{i} u^{j}-\frac{1}{2} \rho \lambda u^{k} \dot{a}^{3}{ }_{k} g^{i j}-\rho \lambda u^{(i} \dot{a}^{3 j)}+\frac{1}{2} \nabla_{k}\left[u^{i} \Sigma^{j) k}\right] \\
& +\frac{1}{2} u^{k} \nabla_{k}\left[\lambda u^{(i} a^{3 j)}\right]-\rho k a^{(1 i} \dot{a}^{2 j)}-\frac{1}{2} \rho k a^{1 k} \dot{a}^{2}{ }_{k} g^{i j}-\frac{1}{2} \nabla_{k}\left[S^{k(i} u^{j)}\right]=0 .
\end{aligned}
$$

Inserting equations (17)-(19) and (27) into equation (33) gives after rearranging

$$
\begin{gathered}
\frac{1}{2 \kappa} G^{i j}=\frac{1}{2}\left\{[\rho(1+\epsilon)+p] u^{i} u^{j}+p g^{i j}\right\}+\frac{1}{2} \rho u^{k} \nabla_{k}\left[\lambda u^{(i} a^{3 j)}\right]+\frac{1}{2} u^{(i} S^{j) k} \dot{u}_{k} \\
-\frac{1}{2} \rho \dot{\lambda} u^{(i} a^{3 j)}-\frac{1}{2} T_{S T} u^{i} u^{j}-\rho \lambda u^{(1 i} \dot{a}^{3 j)}+\frac{1}{2} \nabla_{k}\left[u^{(i} \Sigma^{j) k}\right] \\
+\frac{1}{2} \rho u^{k} \nabla_{k}\left[\lambda u^{(i} a^{3 j)}\right]-\frac{1}{2} \nabla_{k}\left[S^{k(i} u^{j)}\right] .
\end{gathered}
$$

All the ' $\lambda$ ' terms in equation (34) can be shown to satisfy the relationship

$$
\begin{gathered}
-\frac{1}{2} \rho u^{k} \nabla_{k}\left[\lambda u^{(i}\right] a^{3 j)}-\frac{1}{2} \rho \dot{\lambda} u^{(i} a^{3 j)}-\rho \lambda u^{(1 i} \dot{a}^{3 j)}+\frac{1}{2} \rho u^{k} \nabla_{k}\left[\lambda u^{(i} a^{3 j)}\right] \\
=-\frac{1}{2} u^{(i} \Sigma^{j) k} \dot{u}_{k}+\frac{1}{2} \nabla_{\ell}\left[u^{\ell} \Sigma^{k(j}\right] u^{i)} u_{k} .
\end{gathered}
$$


At first it would appear that the string energy density term $-\frac{1}{2} T_{S T} u^{i} u$ in equation (34) would renormalize the fluid energy; however, it will be shown below that this is not the case because of the identity

$$
T_{S T} u^{i} u^{j}=-u^{(i} \Sigma^{j) k} \dot{u}_{k}
$$

Therefore substituting equations (35) and (36) into (34) gives the final results for the metric field equation

$$
G^{i j}=\kappa T_{S F}^{i j}
$$

where the spinning string fluid energy-momentum tensor is given by

$$
T_{S F}^{i j}=T_{F}^{i j}+T_{S}^{i j}+T_{S T}^{i j}
$$

where the perfect fluid energy-momentum tensor is

$$
T_{F}^{i j}=[\rho(1+\epsilon)+p] u^{i} u^{i}+p g^{i j}
$$

the spin energy-momentum tensor is

$$
T_{S}^{i j}=u^{(i} S^{j) k} \dot{u}_{k}+\nabla_{k}\left[u^{(i} S^{j) k}\right]
$$

and the string energy-momentum tensor is

$$
T_{S T}^{i j}=\nabla_{k}\left[u^{(i} \Sigma^{j) k}\right]+\nabla_{\ell}\left[u^{\ell} \Sigma^{k(j}\right] u^{i)} u_{k} .
$$

Note that the combination of perfect fluid and spin energy-momentum tensors given by equations (39) and (40) are exactly the Weyssenhoff form for a spin fluid given by Ray and Smalley [11]. In reducing the metric equation to its final form given by equations (37)-(41), we have used the variational equations for the Lagrange multiplier for the fluid continuity and the 4-velocity to obtain the relations (equations (17) and (18))

$$
2 \lambda_{1}=[\rho(1+\epsilon)+p]-T_{S T}
$$

and

$$
\rho \dot{\lambda}_{2}=-[\rho(1+\epsilon)+p]-T_{S T}-T_{S}
$$

respectively. As a result of the extra factor $T_{S T}$ in equation (42), it is quite remarkable that the string energy density $T_{S T}$ does not occur in the fluid energy-momentum tensor given by equation (39). This renormalization does not occur because of the identity given by equation (36) in which the second term, which would have occurred in the string energymomentum tensor, cancels with the left-hand term, which would have occurred in the fluid energy-momentum tensor. No such identity occurs for the spin energy density.

In the next section, the energy-momentum tensor is developed for the case where the energy is considered as a function of the spin and string variables.

\subsection{Extended thermodynamics}

Including the spin [13] and the shear-momenta (twist) [6] as thermodynamic variables has been discussed in earlier works. However, in this section the string variable is also treated as a thermodynamic variable. The energy now becomes

$$
\mathrm{d} \epsilon=T \mathrm{~d} s+\frac{p}{\rho^{2}} \mathrm{~d} \rho+\frac{1}{2} \omega_{i j} \mathrm{~d} s^{i j}+\frac{1}{2} \omega_{i j} \mathrm{~d} \sigma^{i j} .
$$

Since most of the details are a repetition of the previous case, only the salient issues are presented for simplicity. 
3.2.1. Variational equations. Even though the tetrad variations are slightly altered by the extended thermodynamics, the spinning string has the same Fermi-Walker transport properties described in the previous discussions based upon the standard thermodynamics. The changes in the metric field equation are given in the next section.

(i) Metric field equation. The metric field equation for the extended thermodynamics now becomes

$$
G^{i j}=\kappa T_{S F}^{i j}
$$

where the improved $T_{S F}^{i j}$ is now the sum of a perfect fluid energy-momentum tensor

$$
T_{F}^{i j}=[\rho(1+\epsilon)+p] u^{i} u^{i}+p g^{i j}
$$

(which is the same as before) the spin energy-momentum tensor is

$$
T_{S}^{i j}=2 u^{(i} S^{j) k} \dot{u}_{k}+\nabla_{k}\left[u^{(i} S^{j) k}\right]-\omega_{k}^{(i} S^{j) k}
$$

and the string energy-momentum tensor is

$$
T_{S T}^{i j}=u^{(i} \Sigma^{j) k} \dot{u}_{k}-\dot{u}^{(i} \Sigma^{j) k} u_{k}+\nabla_{k}\left[u^{(i} \Sigma^{j) k}\right]+\nabla_{\ell}\left[u^{\ell} \Sigma^{k(j}\right] u^{i)} u_{k} .
$$

Equations (46) and (47) are the usual improved spin energy-momentum tensor for a spinning fluid [13]. Note in comparison with the usual Weyssenhoff spin energy-momentum given in equation (40), besides the obvious $\omega \cdot S$ piece, the unusual factor of two for the first term in equation (47).

Before leaving the discussion of the spinning string fluid energy-momentum tensor, an interesting relationship for the temporal development of the string module function can be derived from the 4-momentum variational equation by taking the inner product with the tetrad $a^{3 i}$ to give

$$
\begin{gathered}
-\rho a^{3 i} \nabla_{i} \lambda_{2}+\lambda_{3} a^{3 i} X_{, i}+\lambda_{4} a^{3 i} s_{, i}-\rho \dot{\lambda}-\rho \lambda u_{k} a^{3 i} \nabla_{i} a^{3 k}-\rho k a^{1 k} a^{3 i} \nabla_{i} a_{k}^{2} \\
-\rho \lambda a^{3 i} \omega_{i j} a^{3 j}=0 .
\end{gathered}
$$

Since the last term vanishes, this means that this expression applies to both models treated here. Equation (49) can be rewritten in a more illuminating form by defining the quantities $\lambda_{3}=\rho \hat{\lambda}_{3}, \lambda_{4}=\rho \hat{\lambda}_{4}$ and the spatial gradient along the string $a^{3 i} \nabla_{i} \mu \doteq \dot{\mu}$ so that

$$
\dot{\lambda}+\lambda u_{k} \dot{a}^{3 k}=\dot{\lambda}_{2}+\hat{\lambda}_{3} \dot{X}+\hat{\lambda}_{4} \dot{s}-k a^{1 k} \dot{a}_{k}^{2}
$$

which show explicitly the strong dependence of the time development of the string module function on the gradients of the matter along the string.

\section{Application to spinning strings}

The above discussion describes a universe populated by a spinning string fluid (in contrast to the dust model described by Letelier $[3,16]$ and the fluid models described in paper I). However, to understand the content of this paper, it is necessary to briefly recall the dust model as a basis for extending the concept to spinning string systems. This is described in the application below which then extends a static, spherically symmetric, bi-vector string array in general relativity (without spin). 


\subsection{Extended fluid model—no spin}

The stress-energy tensors discussed in I give exact solutions for a static, spherically symmetric, bi-vector string array in GR previously studied by Letelier [3,14-16] and Soleng [17]. The starting point for their calculation is the hypothesized string stress-energy tensor

$$
T_{i j}=\Sigma_{i}{ }^{k} \Sigma_{j k}
$$

where for simplicity, a density and a normalization factor have been absorbed into the definition of the string bi-vector which is defined as

$$
\kappa \Sigma_{j k}=\frac{1}{2} S_{[j} u_{k]} .
$$

The connection to equation (3) is given by

$$
S^{j}=-4 \kappa \rho \lambda(x) a^{3 j} .
$$

Letelier studied both string clouds [3] and string fluids [16]. One of the applications, discussed in I, of the static string fluid formalism was an extension of the Schwarzschild vacuum solution to a black hole surrounded by a fluid of strings. The static solution is

$\mathrm{d} s^{2}=-\left(1-h-\frac{2 M}{r}\right) \mathrm{d} t^{2}+\frac{\mathrm{d} r^{2}}{1-h-2 M / r}+r^{2} \mathrm{~d} \theta^{2}+r^{2} \sin ^{2} \varphi \mathrm{d} \theta^{2}$

where $M$ is a constant. In paper I, the symbol $a$ was used. To avoid confusion with the Kerr parameter $a$, the parameter $h$ will be used here. In this extension, the string fluid has zero pressure, and the string function $S^{j}$ is radial given by

$$
S^{j}=\left(0,-\frac{4 h}{b M}, 0,0\right)
$$

where the thermodynamic parameter $b$ for the string is defined in the following section. The density of the fluid is then given by

$$
\rho=-\frac{2 h}{b M r}\left(1-h-\frac{2 M}{r}\right) \text {. }
$$

The requirement of positive density requires $h$ to be negative and the presence of the string fluid around the black hole causes the horizon to contract away from its Schwarzschild value. In the next section, the static solution described by equations (54)-(78) is extended to a stationary spacetime fluid with spin.

\subsection{Extension to spinning string fluid}

4.2.1. The metric. The application of spinning strings described here is a slow-rotation solution to the static Schwarzschild system described above. The stationary metric used is given by

$$
\mathrm{d} s^{2}=-f \mathrm{~d} t^{2}-2 k(r, \theta) \mathrm{d} \varphi \mathrm{d} t+\frac{\mathrm{d} r^{2}}{f}+r^{2} \mathrm{~d} \theta^{2}+r^{2} \sin ^{2} \theta \mathrm{d} \varphi^{2}
$$

where the metric function $k$ is only a function of the $r, \theta$ coordinates. It is possible that a particular solution of the metric could have the same form as in equation (53), but that is not a requirement. 
4.2.2. The spin-string contributions. The possible spin tensor contributions are $S_{r \varphi}, S_{\varphi \theta}$ and $S_{r \theta}$. Only the case of a $\theta$ component of the spin vector is considered in this work; so the spin tensor components $S_{\varphi \theta}$, and $S_{r \theta}$ are taken to be zero. Only the specific case where the spin density $S_{r \varphi}$ is proportional to the fluid vorticity will be studied in this application. Thus

$$
S_{r \varphi}=A \frac{f k_{, r}-k f_{, r}}{f \sqrt{f}}
$$

where $A$ is a constant and the subscript ' $r$ ' is the partial derivative with respect to $r$.

The spin variables, like the string variable, may or may not be a thermodynamic variable with respect to the second law. To indicate this possibility, the parameter $b$ (as in I) refers to the string thermodynamics properties, and here, the parameter $\bar{b}$ refers to the spin, so that

$$
\begin{array}{ll}
b=1 \text { and/or } \bar{b}=1 & \text { variables are not thermodynamic } \\
b=2 \text { and/or } \bar{b}=2 & \text { variables are thermodynamic. }
\end{array}
$$

Given this parametrization, the string fluid contributions from equations (41) and (48) are given by

$\kappa T_{S T}^{i j}=\frac{1}{4}\left\{-u^{i} u^{j}\left(S_{; k}^{k}+b S^{k} \dot{u}_{k}\right)+b \dot{u}^{(i} S^{j)}+2 u^{(i} \dot{S}^{j)}+2 u^{(i} S^{j)} u_{; k}^{k}-S^{k} \nabla_{k}\left(u^{i} u^{j}\right)\right\}$

and the spin fluid contributions from equations (40) and (47) are given by

$$
T_{S}^{i j}=\bar{b} u^{(i} S^{j) k} \dot{u}_{k}+u^{(i} W^{j)}-\left\{S^{k(i}\left[u^{j)}{ }_{; k}+(\bar{b}-1) \omega^{j)}{ }_{k}\right]\right\}
$$

where the divergence of the spin density is given by

$$
W^{j}=\nabla_{k} S^{j k} .
$$

Note that the factor $\kappa$ appears in equation (61) for the string energy-momentum since the definition of the string function equation (53) is defined explicitly in terms of $\kappa$.

\subsection{The field equations for spinning string fluids}

The only relevant field equation that must be considered in detail is a non-zero off-diagonal equation. In the appendix, the non-zero stress-energy components associated with the spinning string fluid are the diagonal terms and the two off-diagonal terms $T_{r \theta}$ and $T_{(0 \varphi)}$ where the parentheses enclosing the coordinates indicate that they refer to the non-holonomic coordinates associated with the set of tetrads that are used to diagonalize the metric. The diagonal stress-energy tensors have a spin contribution proportional to the product of the spin and the vorticity. The slow-rotation approximation is defined by the expansion of all terms to first order in $k$ and its derivatives which are also expanded to first order in the Kerr parameter $a$. Thus in the slow-rotation approximation, these terms will not contribute to the diagonal stress-energy tensors, and therefore their solution will be the same as in the static case. The same is true of the off diagonal stress-energy component $T_{r \theta}$. Thus the basic solution for the slowly rotating black hole with a spinning string fluid will be the same as in the static case. There will be no pressure and the energy density and string density will be the same. However, the rotation of the black hole and the spin of the string system enter through the $G_{(0 \varphi)}$ field equation. At first, this may seem to be a trivial addition to the static case; however, the addition of spin to the system produces significant changes in the metric parameter ' $h$ '. 
4.3.1. The $G_{(0 \varphi)}$ field equation. The $G_{(0 \varphi)}$ equation obtained is given by

$$
\begin{gathered}
{\left[\left(\frac{f k_{, r}-k f_{, r}+f \sqrt{f} S_{\varphi r}}{D}\right) \mathrm{e}^{(\beta-\mu) / 2}\right]_{, r}+\left[\left(\frac{f k_{, \theta}-k f_{, \theta}+f \sqrt{f} S_{\varphi \theta}}{D}\right) \mathrm{e}^{(\beta-\mu) / 2}\right]_{, \theta}} \\
=\mathrm{e}^{(\beta-\mu) / 2} \frac{\sqrt{f} S_{\varphi}^{k} f_{, k}(1-\bar{b})}{D}
\end{gathered}
$$

where the function $D$ is given by

$$
D^{2}=f r^{2} \sin ^{2}(\theta)+k^{2} \approx f r^{2} \sin ^{2}(\theta)
$$

to first order in $k$. Upon substituting the chosen spin values, the $G_{(0 \varphi)}$ field equation becomes

$$
(A+1)\left(\frac{f k_{, r}-k f_{, r}}{\sin \theta}\right)_{, r}+\left(\frac{k_{, \theta}}{r^{2} \sin \theta}\right)_{, \theta}=\left(\frac{f_{, r}(1-\bar{b})}{f \sin \theta}\right) A\left(f k_{, r}-k f_{, r}\right) .
$$

The $k$ value for the full Kerr metric, to first order in the Kerr parameter $a$, is variable and proportional to $\sin ^{2}(\theta)$. Making the same choice here gives

$$
k=K(r) \sin ^{2}(\theta) \text {. }
$$

Substituting equation (66) into (65) then yields

$$
(A+1)\left(f K_{, r}-K f_{, r}\right)_{, r}-\frac{2 K(r)}{r^{2}}=A \frac{f, r}{f}\left(f K_{, r}-K f_{, r}\right)(1-\bar{b})
$$

which has the solution of the form

$$
K(r)=f r^{n}, \quad n \neq 0,1
$$

with the constraints

$$
\begin{aligned}
& (A+1)(3-n)=A(1-\bar{b}) \\
& (A+1) n(n-1)(1-h)=2 .
\end{aligned}
$$

From this, it can be immediately seen whether or not the spin being a thermodynamic variable is theoretically important in setting the range of possible solution parameters.

4.3.2. Spin is not a thermodynamic variable. If the spin is not a thermodynamic variable, then the spin parameter $\bar{b}=1$. From equation (69), it is only possible to have the value of $n=3$. Thus there are unique relationships between the $A$ parameter (the constant of proportionality between the spin density and the vorticity) and the metric parameter $h$ given by

$$
\begin{aligned}
& 1-h=\frac{1}{3(A+1)}, \\
& k=f r^{3} \sin ^{2}(\theta),
\end{aligned}
$$

and

$$
\sqrt{f} S_{\varphi r}=3 A r^{2} \sin ^{2}(\theta)
$$


4.3.3. Spin is a thermodynamic variable. If the spin is a thermodynamic variable, then the spin parameter $\bar{b}=2$. There is now a family of equations parametrized by $n$. For each $n$, there is a single choice of $A$ and $h$ such that

$$
\begin{aligned}
& A=\frac{n-3}{4-n} \\
& 1-h=\frac{2(4-n)}{n(n-1)} \\
& k=f r^{n} \sin ^{2}(\theta) \\
& \sqrt{f} S_{\varphi r}=A n r^{n-1} \sin ^{2}(\theta) .
\end{aligned}
$$

The functional relationship between $h$ and $n$ given by equation (74) will be important in the discussions given below.

Since the other field equations in the slow-rotation limit are unchanged from their static values, then the fluid parameters must also have the same forms

$$
\begin{aligned}
& S^{j}=\left(0,-\frac{4 h}{b M}, 0,0\right) \\
& \rho=-\frac{2 h}{b M r}\left(1-h-\frac{2 M}{r}\right) .
\end{aligned}
$$

It can clearly be seen that the metric parameter $h$ must be negative in order to get positive energy density. From equation (74), the values of $n$ corresponding to negative $h$ are limited to the ranges

$$
1<n<2.37 \quad \text { and } \quad-3.37<n<0 .
$$

Thus the presence of spin has restricted the possible values of $h$. Note that in this model, no spin corresponds to the single value $n=3$ which is out of the parameter range necessary for positive density. Also the parameter $A$ is always negative in the above ranges since the spin opposes the vorticity.

\section{Discussions and conclusions}

We have developed a stress-energy tensor describing a spinning string fluid. The string fluid can potentially have mass, stress and spin content. Although the theoretical framework for the spinning string fluid is derived in the Riemannian spacetime of GR, the form of energy-momentum tensor is in general valid for four cases: general relativity and the Einstein-Cartan theory, with and without the spin treated as a thermodynamic variable. The demonstration of the case of the EC theory in RC spacetime, which uses the extended connection of RC spacetime, will be shown in a future work.

The application made here is the extension of a Schwarzschild black hole from a static to a slowly rotating black hole in a stationary spacetime in GR. The off-diagonal metric is then parametrized in terms of a parameter $n$. The enlarged parameter content of the fluid makes this approach a valuable tool for modelling the interactions of string structures in stationary spacetimes. If, for example, the spin is not a thermodynamic variable, then $n=3$, and there is a unique relationship among the spin, the vorticity and the metric parameter $h$ which determines the radius of the event horizon of the black hole. However, the vorticity parameter must satisfy $A \leqslant-\frac{2}{3}$ in order to ensure positive densities. The case $A=-\frac{2}{3}$ just gives the Schwarzschild radius for the rotating black hole since then the metric parameter $h=0$. 
When the spin is treated as a thermodynamic variable, then equation (74) shows that there is a family of solutions described by a range of possible $n$ values which give both positive densities and spin.

The examples above shows that the extension of a black hole to a slowly rotating black hole emphasizes the necessity of spin to maintain a positive mass density. In all cases described above, the spin of the fluid opposes the vorticity in the region around the black hole. Since equation (78) requires $h<0$ for positive density, then the event horizon is contracted by the presence of the string system (except for the case $n=3$ ). The amount of the contraction is determined by the spin.

\section{Appendix}

The general spacetime metric is given by

$$
\mathrm{d} s^{2}=-f \mathrm{~d} t^{2}-2 k \mathrm{~d} t \mathrm{~d} \varphi+\mathrm{e}^{\mu} \mathrm{d} r^{2}+\mathrm{e}^{\beta} \mathrm{d} \theta^{2}+\frac{D^{2}-k^{2}}{f} \mathrm{~d} \varphi^{2}
$$

where $f, k, \mu, \beta$ and $D$ are considered to be functions of $r$ and $\theta$. A convenient set of tetrads for calculational purposes (not to be confused with the set of tetrads discussed in section 1) that diagonalize the metric is given by

$$
\begin{aligned}
-u_{i} \equiv A^{(0)}{ }_{i} & =(\sqrt{f}, 0,0, k / \sqrt{f}) \\
A^{(1)}{ }_{i} & =\left(0, \mathrm{e}^{\mu / 2}, 0,0\right) \\
A^{(2)}{ }_{i} & =\left(0,0, \mathrm{e}^{\beta / 2}, 0\right) \\
A^{(3)}{ }_{i} & =(0,0,0,-D / \sqrt{f}) .
\end{aligned}
$$

The string vector has the components

$$
S=\left(0, S_{r}, S_{\theta}, 0\right) \text {. }
$$

The tetrad indexed diagonal components of the stress-energy tensor are given by

$$
\begin{aligned}
& \kappa T_{(00)}=\rho-\frac{1}{4} S_{; k}^{k}+\frac{2-b}{8 f} f_{, a} S^{a}-\frac{W_{0}}{\sqrt{f}} \\
& \kappa T_{(11)}=t_{(11)}+\frac{b}{8 f} f_{, r} S^{r}+\bar{b} \frac{S_{\varphi}^{r}}{2 \sqrt{f} D^{2}}\left[k f_{, r}-f k_{, r}\right] \\
& \kappa T_{(22)}=t_{(22)}+\frac{b}{8 f} f_{, \theta} S^{\theta}+\bar{b} \frac{S_{\varphi}{ }^{\theta}}{2 \sqrt{f} D^{2}}\left[k f_{, \theta}-f k_{, \theta}\right] \\
& \kappa T_{(33)}=t_{(33)}+\bar{b} \frac{S_{\varphi}{ }^{a}}{\sqrt{f} D^{2}}\left[k f_{, a}-f k_{, a}\right]
\end{aligned}
$$

where the summation index $a=r, \theta$. Then the off-diagonal stress-energy components are given by

$$
\begin{aligned}
& \kappa T_{(0) r}=0 ; \quad \kappa T_{r(3)}=+b \frac{f_{, r} S_{\varphi}}{16 \sqrt{f} D} \\
& \kappa T_{(0) \theta}=0 ; \quad \kappa T_{\theta(3)}=b \frac{f_{, \theta} S_{\varphi}}{16 \sqrt{f} D} \\
& \kappa T_{(03)}=-\frac{1}{2 \sqrt{f} D}\left(k W_{0}-f W_{\varphi}\right)-\frac{S_{\varphi}{ }^{a} f_{, a}}{4 \sqrt{f} D^{2}}(1-2 \bar{b}) ; \\
& \kappa T_{r \theta}=\frac{b}{8 f} f_{,(r} S_{\theta)}-\bar{b} \frac{S_{\varphi(\theta}}{2 \sqrt{f} D^{2}}\left(f k_{, r}-k f_{, r)}\right) .
\end{aligned}
$$




\section{References}

[1] Zeldovich Ya B 1980 Mon. Not. R. Astron. Soc. 192663

[2] Vilenkin A 1981 Phys. Rev. Lett. 461169

Vilenkin A 1981 Phys. Rev. Lett. 461496 (erratum)

[3] Letelier P S 1979 Phys. Rev. D 201294

[4] Smalley L L and Krisch J P 1996 Class. Quantum Grav. 13 L19

[5] Halbwachs F 1960 Theorie Relativiste des Fluide à Spin (Paris: Gauthier-Villars)

[6] Smalley L L and Krisch J P 1995 J. Math. Phys. 36778

[7] Smalley L L and Krisch J P 1997 Class. Quantum Grav. 142405

[8] Ray J R 1972 J. Math. Phys. 131451

[9] Nieto J A 1995 Mod. Phys. Lett. A 103087

[10] Krisch J P 1996 Gen. Rel. Grav. 2869

[11] Ray J R and Smalley L L 1982 Phys. Rev. D 262619

[12] Ray J R, Smalley L L and Krisch J P 1987 Phys. Rev. D 353261

[13] Ray J R and Smalley L L 1982 Phys. Rev. Lett. 491059

Ray J R and Smalley L L 1982 Phys. Rev. Lett. 50525 (erratum)

[14] Letelier P S 1980 Phys. Rev. D 22807

[15] Letelier P S 1981 Nuovo Cimento B 63519

[16] Letelier P S 1983 Phys. Rev. D 282414

[17] Soleng H H 1995 Gen. Rel. Grav. 27367 